\title{
RESOLUTION OF A HIGH PERFORMANCE CAVITY BEAM POSITION MONITOR SYSTEM*
}

\author{
S. Walston ${ }^{\dagger}$, C. Chung, P. Fitsos, J. Gronberg, LLNL, Livermore, CA, USA \\ M. Ross, FNAL, Batavia, IL, USA \\ O. Khainovski, Y. Kolomensky, P. Loscutoff, LBNL, Berkeley, CA, USA
}

M. Slater, M. Thomson, D. Ward, University of Cambridge, Cambridge, UK

S. Boogert, Royal Holloway, University of London, Egham, UK

V. Vogel, DESY, Hamburg, Germany

R. Meller, Cornell University, Ithaca, NY, USA

A. Lyapin, S. Malton, D. Miller, University College London, London, UK

J. Frisch, S. Hinton, J. May, D. McCormick,

S. Smith, T. Smith, G. White, SLAC, Menlo Park, CA, USA

T. Orimoto, Caltech, Pasadena, CA, USA

H. Hayano, Y. Honda, N. Terunuma, J. Urakawa, KEK, Tsukuba-shi, Ibaraki-ken, Japan

\section{Abstract}

International Linear Collider (ILC) interaction region beam sizes and component position stability requirements will be as small as a few nanometers. It is important to the ILC design effort to demonstrate that these tolerances can be achieved - ideally using beam-based stability measurements. It has been estimated that RF cavity beam position monitors (BPMs) could provide position measurement resolutions of less than one nanometer and could form the basis of the desired beam-based stability measurement. We have developed a high resolution RF cavity BPM system. A triplet of these BPMs has been installed in the extraction line of the KEK Accelerator Test Facility (ATF) for testing with its ultra-low emittance beam. A metrology system for the three BPMs was recently installed. This system employed optical encoders to measure each BPM's position and orientation relative to a zero-coefficient of thermal expansion carbon fiber frame and has demonstrated that the three BPMs behave as a rigid-body to less than $5 \mathrm{~nm}$. To date, we have demonstrated a BPM resolution of less than $20 \mathrm{~nm}$ over a dynamic range of $+/-20$ microns.

\section{THEORY}

When a bunch transits a cavity BPM, the field of the bunch excites the eigenmodes of the electromagnetic fields within the cavity. The amplitude of the $\mathrm{TM}_{110}$ mode has a linear dependence on the transverse offset of the beam rela-

\footnotetext{
${ }^{*}$ This work was performed under the auspices of the U.S. Department of Energy by Univeristy of California Lawrence Livermore National Laboratory under contract No. W-7405-Eng-48, by University of California Lawrence Berkeley National Laboratory under contract No. DE-FG0203ER41279, and by the Stanford Linear Accelerator Center under contract No. DE-AC02-76SF00515. This work was supported by the National Science Foundation, by the Japan-USA Collaborative Research Grant, Grantin-Aid for Scientific Research from the Japan Society for the Promotion of Science, and by the Commission of the European Communities under the 6th Framework Programme "Structuring the European Research Area," contract number RIDS-011899.

†walston2@1ln1.gov
}

06 Instrumentation, Controls, Feedback \& Operational Aspects tive to the electrical center of the cavity; the phase depends on the direction of the offset. The $\mathrm{TM}_{110}$ mode also has a linear dependence on both the angle of attack and angle of obliquity (collectively referred to as "tilt") of a finite length bunch relative to the $z$-axis of the cavity. This is discussed in more detail elsewhere $[1,2,3]$.

The intrinsic resolution of a BPM is limited by the signal to noise ratio of the system: The signal voltage of the BPM is determined by the beam's energy loss to the $\mathrm{TM}_{110}$ mode and by the external coupling of the waveguide; the overall noise of the system comes from thermal noise as well as contamination from the symmetric $\mathrm{TM}_{010}$ mode. It has been estimated that an RF cavity BPM could have a resolution below one nanometer [4].

\section{EXPERIMENT}

This experiment employed three identical cavity BPMs [5]. Single bunch extractions from the ATF ring of typically between 6 and $7 \times 10^{9} e^{-}$at an energy of $1.28 \mathrm{GeV}$ were used for our tests. The machine repetition rate was $\sim 1 \mathrm{~Hz}$.

Because the beam passed through the apparatus in a straight line, the beam's position in BPM 2 was related in a linear way to the beam's positions in BPMs 1 and 3. BPM resolution was determined by measuring the residual - that is the difference between the position of the beam as measured by BPM 2 and the predicted position as calculated from the beam's parameters measured by BPMs 1 and 3. The coefficients used to calculate the beam's position at BPM 2 were determined by regressing the beam's $y$ position measured by BPM 2, $y_{2}^{\text {beam }}$, against $x_{1,3}^{\text {beam }}, y_{1,3}^{\text {beam }}$, $x_{1,3}^{\prime \text { beam }}$ and $y_{1,3}^{\prime \text { beam }}$ over many events. The resolution was then proportional to the standard deviation of the distribution of the residuals for many ATF extractions.

Details of the experiment and explanations of the waveform processing, calibration, and resolution algorithms are all discussed in nauseating detail elsewhere [3].

T03 Beam Diagnostics and Instrumentation 


\section{RESOLUTION STABILITY}

The resolution of the BPM system had previously been measured at $15.6 \mathrm{~nm}$ for position and $2.1 \mu \mathrm{rad}$ for tilt [3]. On 5 December 2006, 17:21 JST, a long test was conducted to investigate the stability of the resolution of the BPM system. A total of 25,600 events were collected over a period of $\sim 4.5$ hours. Using nominally 1024 events per regression (cuts were imposed to remove ATF extractions outside the dynamic range of the system) yielded 25 separate measurements of the resolution each spanning $\sim 11$ minutes. The mean resolution was $18.1 \pm 1.4 \mathrm{~nm}$ while the best resolution was $15.3 \mathrm{~nm}$. Figure 1 shows the stability of the system on the time scale of several hours.

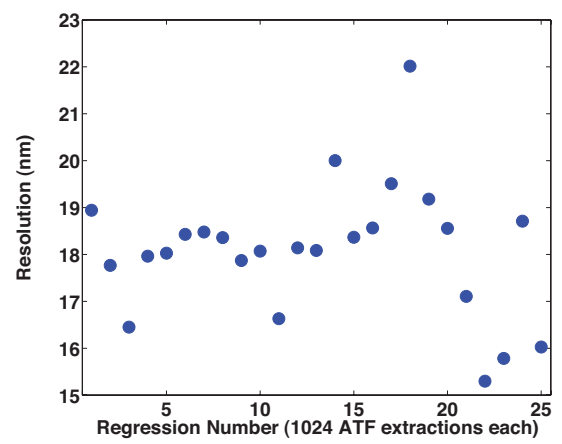

Figure 1: The mean resolution was determined to be $18.1 \pm 1.4 \mathrm{~nm}$ using 25,600 events over $\sim 4.5$ hours from 5 December 2006, 17:21 JST.

\section{METROLOGY SYSTEM}

A metrology system, installed in January 2006, was intended to make possible an evaluation of the non-rigidbody mechanical motion among the three BPMs and especially that part due to thermal drifts.

Three NanoGrid Model A Hi-Resolution systems, manufactured by Optra Inc. were mounted on each BPM [6]. The Optra NanoGrid is an $x y$ metrology system that measures the position of a sensor head relative to an optical encoder grid consisting of a 2-dimensional, 10 micron pitch diffraction grating on soda-lime glass. Each sensor head, which contained a diode laser source, imaged the two interference patterns onto 90-element triple detector arrays to make very accurate phase measurements. When the encoder grid moved relative to the sensor head, the fringes moved across the detector arrays generating $R, S$ and $T$ signals which were $120^{\circ}$ apart in phase. These three signals made possible a phase measurement which was independent of the laser power, the reflectivity of the grid, and the relative intensities in the \pm 1 diffracted orders. This approach made possible a shot-noise-limited phase resolution of 1 part in $2^{14}$ corresponding to a measurement resolution of $0.305 \mathrm{~nm}$.

By employing three NanoGrids per BPM, the $x, y, z$, yaw $\psi$, pitch $\theta$, and roll $\phi$ of each BPM could be pre06 Instrumentation, Controls, Feedback \& Operational Aspects

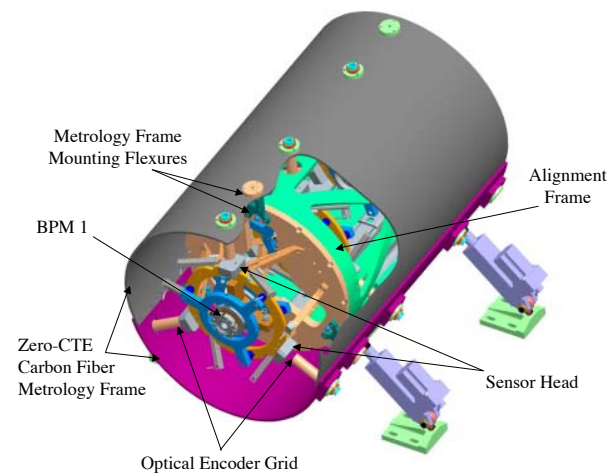

Figure 2: The NanoBPM experiment including the metrology system.

cisely determined [7]. A zero-coefficient of thermal expansion (zero-CTE) carbon fiber metrology frame supported all nine encoder grids. This is shown in Figure 2.

The vibrational modes of the experiment were determined with an acoustic tone generator. The non-rigid-body motion induced in the experiment was then measured by the metrology system and is plotted against frequency in Figure 3. The first vibrational mode of the BPM alignment frame was previously measured at $\sim 190 \mathrm{~Hz}$.

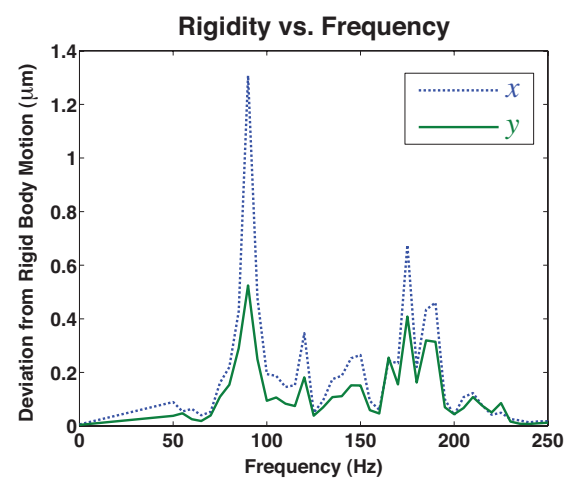

Figure 3: Non-rigid-body motion measured by the metrology system as a function of frequency for both the $x$ and $y$ directions.

For the data of 5 December 2006, 17:21 JST, the degree of non-rigid-body motion measured by the metrology system was $6.0 \pm 0.3 \mathrm{~nm} x$ and $4.1 \pm 0.2 \mathrm{~nm} y$ (this naturally included contributions from both the BPMs and the metrology frame itself).

An enclosure of Dynamat Dynil — a dense acoustic barrier - was subsequently installed around the experiment [8]. Dynil is advertised to have a transmission loss of $\sim 18$ $\mathrm{dB}$ for frequencies less than $250 \mathrm{~Hz}$. After installation of the Dynil acoustic barrier, non-rigid-body motion measured by the metrology system reduced to $5.3 \pm 0.2 \mathrm{~nm} x$ and $3.5 \pm 0.3 \mathrm{~nm} y$. This is shown in Figure 4.

With the metrology system, $y_{2}^{\text {beam }}$ could be regressed T03 Beam Diagnostics and Instrumentation 


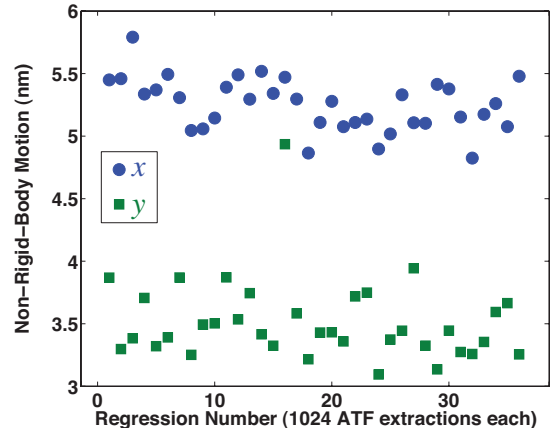

Figure 4: Non-rigid-body motion for both the $x$ and $y$ directions measured by the metrology system over a period of $\sim 6.5$ hours on 6 December 2006 starting at 23:59 JST.

against not only $x_{1,3}^{\text {beam }}, y_{1,3}^{\text {beam }}, x_{1,3}^{\prime \text { beam }}$ and $y_{1,3}^{\prime \text { beam }}$ as ordinarily, but in addition the positions of the three BPMs, $x_{1,2,3}^{\mathrm{BPM}}, y_{1,2,3}^{\mathrm{BPM}}, z_{1,2,3}^{\mathrm{BPM}}, \psi_{1,2,3}^{\mathrm{BPM}}, \theta_{1,2,3}^{\mathrm{BPM}}$, and $\phi_{1,2,3}^{\mathrm{BPM}}$ as measured by the metrology system. For the data of 5 December 2006, 17:21 JST, the mean difference in resolution from including the parameters measured by the metrology system was $-0.2 \pm 0.1 \mathrm{~nm}$, shown in Figure 5. This level of improvement was consistent with $2.7 \mathrm{~nm}$ of non-rigidbody motion among the BPMs. If the measured non-rigidbody motion of $4.1 \pm 0.2 \mathrm{~nm}$ was considered to be coming equally from both the metrology frame and the BPMs, it would imply that there was $2.9 \mathrm{~nm}$ of non-rigid-body motion among the BPMs.

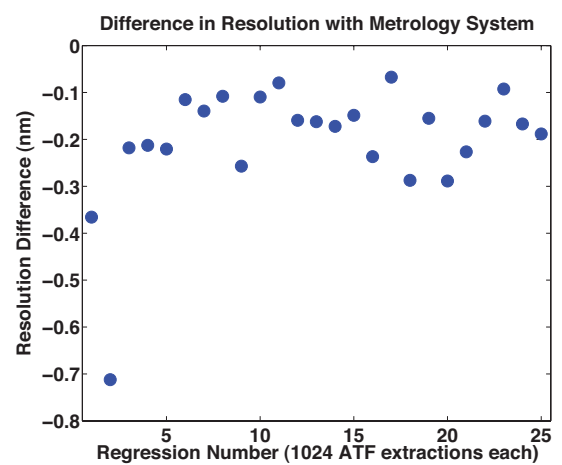

Figure 5: Difference in resolution when the positions of the three BPMs as measured by the metrology system were added to the regression for the beam's $y$ position in BPM $2, y_{2}^{\text {beam }}$. The mean difference was $-0.2 \pm 0.1 \mathrm{~nm}$.

\section{CONCLUSIONS}

To date, the BPM system achieved a position resolution of $15.3 \mathrm{~nm}$, or $15.1 \mathrm{~nm}$ when the parameters measured by the metrology system were included in the regression for $y_{2}^{\text {beam }}$. Over a period of $\sim 4.5$ hours, the system achieved a mean resolution of $18.1 \pm 1.4 \mathrm{~nm}$ - indicative of the degree of stability on that time scale.

06 Instrumentation, Controls, Feedback \& Operational Aspects

\begin{tabular}{|l|c|}
\hline Best measured resolution & $15.3 \mathrm{~nm}$ \\
\hline \hline Mechanical non-rigid-body-motion & $2.7 \mathrm{~nm}$ \\
\hline Thermal noise (at $T=293 \mathrm{~K})$ & $2.8 \mathrm{~nm}$ \\
\hline Phase noise $(\sim 2.5 \mathrm{mrad})$ & $1.3 \mathrm{~nm}$ \\
\hline $\begin{array}{l}\text { Magnetic field fluctuations } \\
(d B=120 \mathrm{nT}, \text { assuming } \vec{B} \text { along } \hat{x})\end{array}$ & $1.3 \mathrm{~nm}$ \\
\hline $\begin{array}{l}\text { Energy jitter } \\
(d E / E=0.0002, \text { assuming } \vec{B} \text { along } \hat{x})\end{array}$ & $0.07 \mathrm{~nm}$ \\
\hline \hline Total measured contributions & $4.3 \mathrm{~nm}$ \\
\hline \hline $\begin{array}{l}\text { Contributions to resolution } \\
\text { remaining unaccounted for }\end{array}$ & $14.7 \mathrm{~nm}$ \\
\hline
\end{tabular}

Table 1: Contributions to the measured resolution.

The various known contributions to the resolution are listed in Table 1. The metrology system has now ruled out mechanical non-rigid-body-motion as a significant contributor to the measured resolution. The bulk of the resolution thus remains unexplained. However, given the poor machining tolerances of these cavities (e.g. roundness of the beam pipe surfaces of the BPMs ranged from 44 to $240 \mu \mathrm{m})$, two important effects have not been ruled out: cross talk between the $x$ and $y$ channels where signal resulting from the beam's offset in $x$ contaminates the signal from the beam's offset in $y$, and contamination from the monopole mode leaking through imperfect coupling slots. Considering the relative sizes of the monopole $\mathrm{TM}_{010}$ and $\mathrm{TM}_{020}$ modes as compared to the $\mathrm{TM}_{110}$ mode, the latter effect was likely more important.

\section{REFERENCES}

[1] D. H. Whittum and Y. G. Kolomensky, Rev. Scientific Instr. 70 (1999), 2300.

[2] Alexey Lyapin, "Strahllagemonitor fuer das TESLAEnergiespektrometer," Ph.D. Dissertation, TU-Berlin, Berlin, 2003.

[3] S. Walston et al., "Performance of a high resolution cavity beam position monitor system," to be published in Nucl. Instr. and Meth. A (2007). SLAC-PUB-12492.

[4] Z. Li, S. R. Smith, T. Naito, and J. Rifkin, Cavity BPM with dipole-mode selective coupler, in: Proceedings of the 2003 Particle Accelerator Conference, Portland, OR, IEEE, Silver Spring, MD, 2003.

[5] V. Balakin et al., "Discussion of nanobeam summaries and areas for future collaboration," http://www.vlepp.serpukhov.su/engl/bnl/bpm.html.

[6] Optra Inc. (http://www.optra.com).

[7] We follow here the mathematical symbols for yaw, pitch, and roll suggested by the American Institute of Aeronautics and Astronautics (AIAA) and the American National Standards Institute (ANSI) in the American National Standard Recommended Practice for Atmospheric and Space Flight Vehicle Coordinate Systems (R-004-1992).

[8] Dynamat Dynil sound barrier (http://www.dynamat.com/products_architectural_dynil.html).

T03 Beam Diagnostics and Instrumentation 